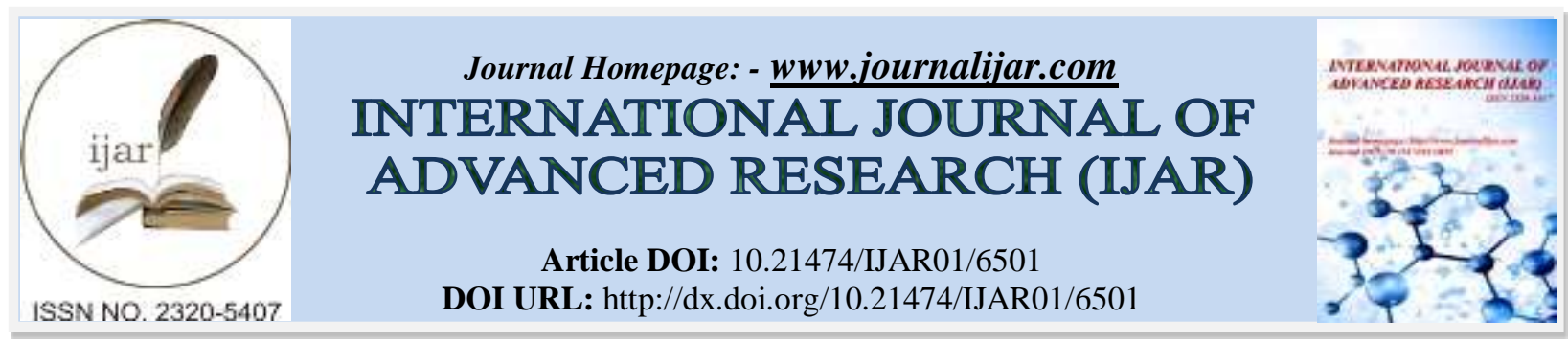

RESEARCH ARTICLE

\title{
CONSEQUENCES OF DOPAMINERGIC AGONIST ASSOCIATED WITH BDNF TREATMENT ON BEHAVIORAL ALTERATIONS AND CYTOLOGICAL CHANGES IN AN EXPERIMENTAL MODEL OF PARKINSON DISEASE DUE TO MANGANESE EXPOSURE.
}

\section{Teresa Ibarra-Gutiérrez ${ }^{1}$, Veronica Anaya-Martínez ${ }^{1}$, Ana L. Gutiérrez-Valdez ${ }^{1}$, José Luis Ordoñez- Librado $^{1}$, Daniel Martínez-Fong ${ }^{2}$, Jorge Aceves-Ruiz ${ }^{2}$, Javier Sanchez-Betancourt ${ }^{1}$, Leonardo Reynoso- Erazo $^{1}$, Enrique Montiel-Flores ${ }^{1}$, Rocío Tron-Alvarez ${ }^{1}$ and ${ }^{*}$ Maria Rosa Avila-Costa ${ }^{1}$. \\ 1. Laboratorio de neuromorfologia, FES iztacala, UNAM, av. De los barrios 1, tlalnepantla 54090, Mexico. \\ 2. Departamento de fisiología, biofisica y neurociencias, cinvestav-ipn, mexico av. Ipn 2508, cp 07360, Mexico.}

\section{Manuscript Info}

........................

Manuscript History

Received: 11 December 2017

Final Accepted: 13 January 2018

Published: February 2018

Key words:-

Manganese inhalation; Parkinson disease experimental model; D3 receptors; 7-OH- DPAT; BDNF.

\section{Abstract}

Parkinson disease (PD) is characterized by dopaminergic (DA) neuronal loss and motor alterations; in this way, an effective therapy should protect and regenerate the DA neurons and striatal innervation. Brain-derived neurotrophic factor (BDNF) is a cell survival mediator and can improve neuronal death, and the activation of DA D3 receptors seems to protect the DA neurons. Thus, there is a synergistic relationship between BDNF and D3 receptors; which would be a neuroprotective therapy. PD animal models, although exhibit some of the features, no one mimics the alterations observed in the disease. PDManganese (Mn) inhalation model was used in this report since it is bilateral, non-invasive and progressive. The rats were exposed to Mn 1 hour 3 times a week, and the motor tests were conducted to measure the performance as well as the progressive damage at 3 and 6 months of exposure. Subsequently, the D3 agonist treatment (7-OH-DPAT) and BDNF gene transfection to DA neurons were co-administered, and then we evaluated its effect measuring the animals' performance, and if the recovery was associated with DA neuronal and striatal dendritic spines preservation. The results showed that the animals presented PD-like motor alterations, and great DA neurons and striatal dendritic spines loss. The behavior recovery was associated with the DA neurons recuperation and with the number of dendritic spines of the striatal neurons. Thus, the BDNF overexpression in DA neurons related to the D3 receptors activation seems to be a promising approach for restoring motor alterations and DA neurons in PD.

Copy Right, IJAR, 2018,. All rights reserved.

\section{Introduction:-}

Parkinson disease (PD) is the second most prevalent neurodegenerative disease, this disorder includes movement abnormalities, such as tremor, rigidity, akinesia, bradykinesia, masked face, postural and gait abnormalities (Naskar et al. 2013) involving a progressive loss of dopaminergic (DA) neurons projecting from the substantia nigra compacta (SNc) to the striatum (Alexander, 2004; Martin, 2011). L-DOPA remains to be the best treatment because it improves most of the motor symptoms; however, it does not prevent the progressive DA neuronal loss and causes, 
after 4-5 years of treatment, dyskinesia (Smith et al. 2012). This suggests that a therapeutic approach preventing neuronal death and promoting growth and regeneration would be a valued approach to control this disease (Martinez-Fong et al. 2012). In this way, several neurotrophic factors have been assessed as potential neuroprotective mediators (Connor and Dragunow, 1998; Cummins and Barker, 2012). Brain-Derived Neurotropic Factor (BDNF) effect on DA neurons is well established (Zhou et al. 1994a; 1994b; Razgado-Hernandez et al. 2015). BDNF is expressed by SNc DA neurons, where it plays a critical role in functions such as cell survival (Hyman et al. 1991), synaptic plasticity (Fritsch et al. 2010), cell proliferation (Im et al. 2010), striatal reinnervation (Yurek et al. 1996), DA release modulation (Blochl and Sirrenberg, 1996; Goggi et al. 2002), DA phenotype induction (Zhou et al. 1994b) and DA D3 receptor expression (Guillin et al. 2001; Razgado-Hernandez et al. 2015). It is also well documented that SNc DA neurons degenerate in the absence of BDNF, suggesting its involvement in PD pathogenesis (Howells et al. 2000; Porritt et al. 2005). The reduced BDNF expression in SNc neurons in PD patients and rats with nigrostriatal DA depletion similarly indicate its participation in the pathogenesis of the disease (Venero et al. 1994; Mogi et al. 1999; Howells et al. 2000; Razgado-Hernandez et al. 2015).

On the other hand, the DA D3 receptor activation also demonstrates trophic effects by increasing the subventricular zone, and neostriatum neurogenesis in adult rat brain via the progenitor cells fast amplification (Van Kampen et al. 2003; Van Kampen and Eckman, 2006); thus, the D3 receptor activation stimulates mitogenesis (Chio et al. 1994; Pilon et al. 1994; Griffon et al. 1997) and increases SNc neuronal dendrites arborization (Collo et al. 2008). Moreover, the D3 receptors selective activation restores the nigrostriatal pathway, improving some motor behavior alterations (Van Kampen and Eckman, 2006; Razgado-Hernandez et al. 2015). Furthermore, it is known that BDNF synthesized by DA neurons is responsible for the onset of D3 receptors during development and to maintain their expression in the adult tissue (Guillin et al. 2001). The activation of these receptors by specific agonists protects neurons from MPTP-induced degeneration. The effect apparently is exerted through BDNF, since blocking BDNF action eliminates such protection ( $\mathrm{Du}$ et al. 2005). This result suggests that there is an interaction between $\mathrm{D} 3$ receptors and BDNF, which would regulate the expression of the D3 receptors, and those would exert their protective effect, and probably its neurogenic effect (Merlo et al. 2011) trough BDNF.

It seems that BDNF facilitates, in part, the DA D3 receptors trophic effect activation (Du et al. 2005) increasing the number of D3 receptors (Guillin et al. 2001; Sokoloff et al. 2002). So, there is the possibility that BDNF potentiates the trophic effect of the activation of dopamine D3 receptors in the adult brain.

Several PD experimental models display many of the distinctive features of the disease; however, none resembles the chronic neurodegenerative features of human PD (Betarbet et al. 2002).

When choosing an animal model for PD, one must consider the amount of similarity or discrepancy between the anatomy, physiology, and behavior between humans and animals. The existing models have been useful for understanding the etiology of the disease and compromise resources for proving new treatments (Potashkin et al. 2010). However, the loss of the nigrostriatal DA pathway that has been replicated in animals, either unilaterally or bilaterally, using a variety of selective toxins or by genetic manipulations, is rapid and not progressive, and for those derived through genetic manipulations relevant to human PD, the loss, although more progressive, may be limited in extent or may not even occur at all (Emborg, 2004).

Recently, we validate a novel PD experimental model in mice (Ordoñez-Librado et al. 2008; 2010a) and rats (Sánchez-Betancourt et al. 2012) through the exposure to the mixture of Manganese (Mn) compounds, Manganese chloride $\left(\mathrm{MnCl}_{2}\right)$ and Manganese acetate $\left(\mathrm{Mn}(\mathrm{OAc})_{3}\right)$ by inhalation. After $\mathrm{Mn}$ mixture inhalation, the rodents presented significant loss of SNc tyrosine hydroxylase (TH)-positive neurons; the loss of these neurons was $67.58 \%$ (Ordoñez-Librado et al. 2008). Later on, we determine whether L-DOPA treatment improves the behavior to ensure that the alterations are of DA origin (Ordoñez-Librado et al. 2010b). In summary, after six months of Mn mixture inhalation, striatal DA content decreased $71 \%$, SNc showed a significant reduction in the number of THimmunopositive neurons, the animals displayed akinesia, postural instability, and action tremor; these alterations were improved with L-DOPA treatment. Our data provided evidence that $\mathrm{MnCl}_{2} / \mathrm{Mn}(\mathrm{OAc})_{3}$ mixture inhalation produces similar morphological, neurochemical and behavioral alterations to those observed in PD, suggesting a useful experimental model for the study of this neurodegenerative disease. Additionally, Mn inhalation is progressive and bilateral, which makes it more reliable. 
Based on this background, and taking into account that BDNF itself also induces neurogenesis from stem cells in the adult brain (Benraiss et al. 2001), we decided to explore whether continuous and chronic administration of the D3 agonist 7-OH-DPAT, associated with BDNF gene transfection to DA neurons recovers the altered motor behavior induced by the inhalation of $\mathrm{MnCl}_{2} / \mathrm{Mn}(\mathrm{OAc})_{3}$, which induces bilateral depletion of the nigrostriatal innervation (Ordoñez-Librado et al. 2008; 2010a; Sanchez-Betancourt et al. 2012), and whether this recovery is associated with innervation restoration.

Thus, the aim of this study was to characterize the motor, and cytological alterations induced by Mn mixture inhalation as PD experimental model and determine if the co-treatment (BDNF gene transfection and D3 agonist (7OH-DPAT)) improves the motor recovery and cell death by means of open field test determining: walking distance (ambulation), rearing and walking speed, freezing time, rotarod performance and bradykinesia and counting the number of TH neurons in the SNc and the number of dendritic spines on striatal medium-sized spiny neurons.

\section{Materials and Methods:- Ethics Statement:}

The experimental protocol was carried out by the National Institute of Health Guide for the Care and Use of Laboratory Animals (NIH Publications No. 80-23) revised 1996; Guide for Care and Use of Laboratory Animals certificated by the Secretaria de Agricultura, Ganadería, Desarrollo Rural, Pesca y Alimentación (SAGARPA) (NOM-062- ZOO-1999, Mexico) and approved by the Institutional Animal Care Committee of UNAM. All efforts were made to minimize the number of animals used and their suffering. Anesthetics (sodium pentobarbital anesthesia, $35 \mathrm{mg} / \mathrm{kg}$, i.p.) was used when appropriate under the direction of a veterinarian to relieve potential pain and distress and was given before euthanasia with the transcardial perfusion with $0.9 \%$ saline, followed by $300 \mathrm{ml}$ paraformaldehyde $4 \%$.

\section{Experimental Protocol:}

Twenty-four male Wistar rats weighing $180 \pm 10 \mathrm{~g}$ were housed in groups of four in hanging plastic cages under controlled light conditions (12/12 h light/dark regime) and fed with Purina Rat Chow (Mexico) and water ad libitum. Body weight was verified daily. The animals were divided into two groups: the first consisted of 18 rats that inhaled the mixture of $\mathrm{MnCl}_{2}, 0.04 \mathrm{M}$ and $\mathrm{Mn}(\mathrm{OAc})_{3}, 0.02 \mathrm{M}$ three times a week for six months to induce the PD model, and the other six were exposed to deionized water under the same conditions (control group) this group was used exclusively for cytological comparison.

Before Mn inhalation, rotarod performance and open field were tested as initial parameters (control condition) of motor coordination, postural balance and muscle rigidity of each rat to track their performance during the experiment. The rats were first evaluated in the rotarod and trained to remain on the rod at 5 and $10 \mathrm{rpm}$ for two min (as described by Razgado-Hernandez et al. (2015)), discarding those animals that following three consecutive days were incapable to stay on the rod. We evaluate the performance of the motor activity in the rotarod (coordination of the legs), in the open field (distance traveled, bradykinesia (walking speed) rearing and freezing), the evaluations were repeated at three and six months while the animals were exposed to Mn mixture. After six months of Mn mixture inhalation, the experimental group $(n=18)$ was divided into subgroups for treatments. One group ( $n=6)$ was treated with continuous administration of D3 agonist (7-OH-DPAT) + BDNF gene transfection; the other group $(n=6)$ was used as a positive control with saline solution treatment. Also, after six months of Mn mixture inhalation, the remaining rats $(n=6)$ were kept for five months without treatment to analyze the number of SNc dopaminergic cells to ensure that there was no cell recovery by stopping Mn inhalation ("recovery group"). No treatment was given to the control group; these animals were maintained for histological comparison.

The D3 receptor agonist 7-OH-DPAT (Sigma, St., Louis, MO) was administered through a micro-diffusion pump (Alzet micro-osmotic pump, model 2006) that was surgically implanted subcutaneously, the pump was activated for 3 months (1mg/day), one week after the pump implantation, transfection of the BNDF (single-shot bilateral and intracerebrally in the $\mathrm{SNc}$ ) was performed. Three months post-implantation motor performance was again tested to determine treatment effects. Motor tests were repeated two months after the pump was removed to determine if the effects were permanent.

At the end of the treatment phase, the rats were sacrificed by transcardial perfusion. The brain tissue containing the $\mathrm{SNc}$ and the striatum were obtained, from which $50 \mu \mathrm{m}$ sections were taken and processed with the 
immunocytochemistry technique for tyrosine hydroxylase (TH) (for the counting of the dopaminergic neurons) and Golgi stain (for the counting of dendritic spines in striatal medium-size spiny neurons) (Figure 1).

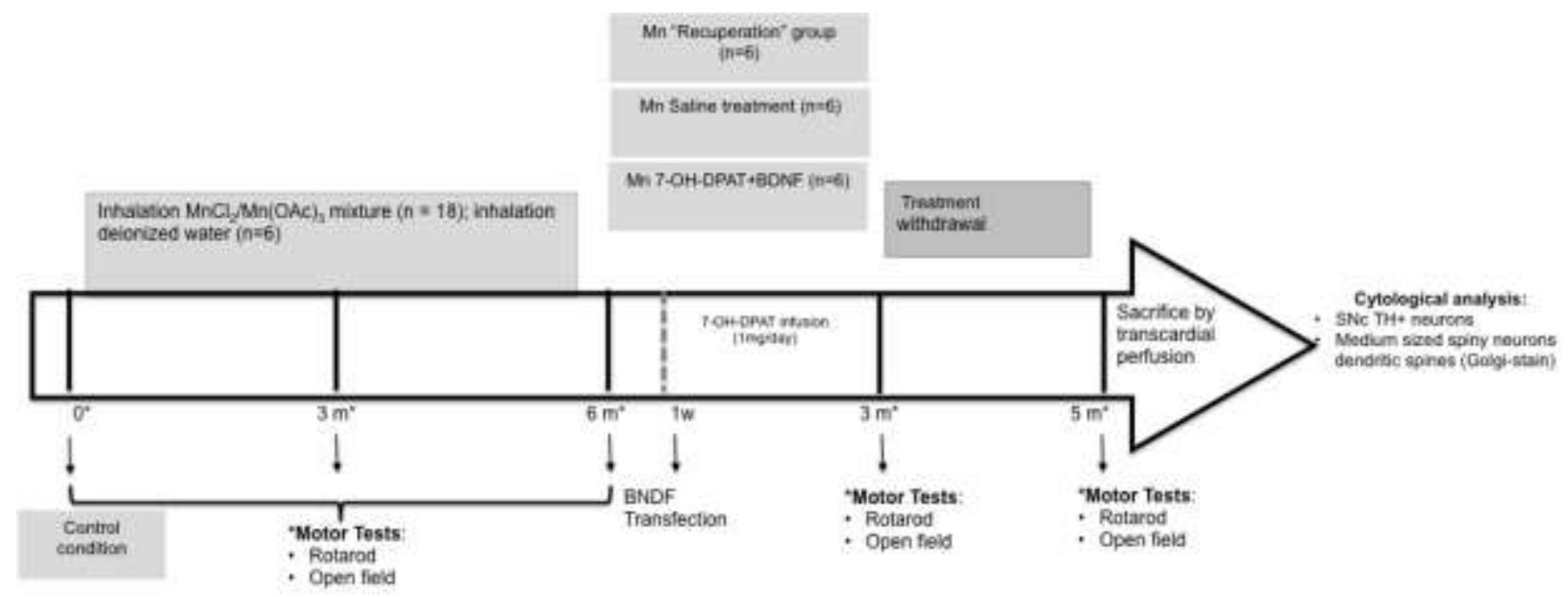

Figure 1:- Experimental design: asterisks indicate motor behavior evaluation, which included rotarod performance and open field activity.

\section{Manganese Inhalation:-}

Inhalations were performed as described by Sánchez-Betancourt et al. (2012), rats were placed in an acrylic chamber inhaling $0.04 \mathrm{M} \mathrm{MnCl}_{2}$ and $0.02 \mathrm{M} \mathrm{Mn}(\mathrm{OAc})_{3}$ one hour three times a week for six months. Control rats inhaled only the vehicle-deionized water-for the same period. Inhalations were implemented in closed acrylic boxes $(40 \mathrm{~cm}$ wide $70 \mathrm{~cm}$ long and $25 \mathrm{~cm}$ high) attached to an ultra-nebulizer (Shinmed, Taiwan), with $10 \mathrm{l} / \mathrm{min}$ continuous flux. The ultra-nebulizer is designed to produce drops in a $0.5-5 \mu \mathrm{m}$ range. A vapor trap is on the opposite side with a solution of sodium bicarbonate to precipitate the remaining metal. During exposures, animals were constantly visually monitored for respiration rate, depth, and regularity. The exposure system was continuously monitored for temperature, oxygen level and Mn concentration (Ordoñez-Librado et al. 2010a; Sánchez-Betancourt et al. 2012).

\section{Motor Tests:-}

\section{Rotarod}

Mn effect on motor coordination was examined by training the rats to remain on a rotarod. It has been established that rodents motor performance, the rotarod allows for the evaluation of the loss or recovery of nigrostriatal innervation in PD rodent models (Diaz et al. 1997; Rozas et al. 1998). As previously described (Razgado-Hernandez et al. 2015), the rotarod contains four-lane rotating rod (diameter $7.5 \mathrm{~cm}$ ) and infrared beams to detect the moment of fall. The body of the animal was placed perpendicular to the rotating axis and the head against the direction of the rotation; the rat must, therefore, move forward to stay on the rod. The rats were trained twice on the rotarod at the constant speed of $5 \mathrm{rpm}$ and $10 \mathrm{rpm}$ for two min during three consecutive days before the first evaluation. In the evaluating phase, the rats were positioned on the rod, and their coordination was evaluated at different speeds $(5,10$, 15, 20 and $25 \mathrm{rpm}$ ) for a maximum of two min at each speed. All animals were video-recorded while remaining on the rod to evaluate the qualitative features of motor coordination and posture (Razgado-Hernandez et al. 2015).

\section{Open Field Test}

The rats were placed in a square compartment of $83 \mathrm{~cm}$ per side. A camcorder recorded the activity for 20 minutes. The obtained videos were analyzed offline to determine 1) the walking distance during $20 \mathrm{~min}$. 2) the total distance traveled during the recording period 3) the dwell time in any area of the field, rearing and walking speed. With these parameters, the relationship between motion and immobility during the recording period and the speed of movement were calculated (Prut and Belzung, 2003). The coordinates of the rat position in the arena were estimated frame by frame from the videos to get the movements' spatiotemporal sequence. Bradykinesia was calculated by the decrease in walking speed judged by the time taken to move from one corner of the arena to the next one, with constant speed. The area was cleaned with a water/alcohol (70\%) solution before every behavioral testing to avoid probable bias due to odors and residues left by rats tested earlier. All experiments were carried out from 11:00 to 15:00 p.m. (Razgado-Hernandez et al. 2015). 


\section{Treatments:-}

\section{D3 Agonist}

The D3 agonist 7-OH-DPAT (Sigma, St., Louis, MO) was systemically administered (Casarrubea et al. 2006) (0.1 $\mathrm{mg} / \mathrm{kg} /$ day) via osmotic pumps (Alzet micro-osmotic pump, model 2006) implanted subcutaneously on the back of the animal. The pumps were implanted under isoflurane anesthesia. The 7-OH-DPAT was infused during three months (1mg/day) (Razgado-Hernandez et al. 2015). One-week post-implantation, BNDF transfection was done, motor assessments were repeated to determine the treatment effects. Motor tests were repeated two months after the pump was removed to determine if the effects were permanent.

\section{BDNF-flag transfection by NTS-polyplex}

The BDNF gene $(2 \mu \mathrm{l})$ was bilaterally transfected into the SNc dopaminergic neurons (the stereotactic coordinates used were $\mathrm{AP}=5 \mathrm{~L}=1.9$ left and -1.9 der $\mathrm{V}=7.1$ from bregma (according to (Paxinos and Watson 2005) a week after initiation D3 receptor agonist administration. The plasmid phDAT-BDNF-flag (10.511 kbp) that codes for BDNF-flag was transfected into the DA neurons using the neurotensin (NTS)-polyplex nanovector (GonzalezBarrios et al. 2006). This transfection is highly selective for DA neurons because the NTS-polyplex nanovector is endocytosed via the high affinity neurotensin receptor present only in the DA neurons (Alvarez-Maya et al. 2001; Hernandez-Baltazar et al. 2012; Martinez-Fong et al. 2012; Razgado-Hernandez et al. 2015). This non-viral transfection method has been shown to be efficient for gene transfection encoding the human BDNF trophic factor to SNc DA neurons (Gonzalez-Barrios et al. 2006; Razgado-Hernandez et al. 2015). Considering that this NTS concentration and the injection volume $(2 \mu \mathrm{L})$, the NTS- polyplex dose at the time of dosing was 2.34 pmol for rats of $550 \mathrm{~g}$ of mean body weight. In accordance with the amount of plasmid DNA, the dose was $419.6 \mathrm{ng}$ of phDATBDNF-flag (Razgado-Hernandez et al. 2015).

\section{Tissue Preparation:-}

Five months after treatment initiation, the animals were sacrificed, anesthetized with sodium pentobarbital lethal dose and perfused via aorta with phosphate buffer saline $(0.1 \mathrm{M} \mathrm{pH} 7.4)$ containing $4 \%$ paraformaldehyde. The brain was removed and placed in the fixative solution for $2 \mathrm{~h}$ and processed for Tyrosine hydroxylase (TH) immunocytochemistry and Golgi stain.

\section{TH Immunocytochemistry}

Coronal sections $(50 \mu \mathrm{m})$ were obtained on a sliding microtome through the mesencephalon for immunohistochemistry. Tyrosine hydroxylase (Chemicon International, Inc. CA, USA, 1: 1000) immunostaining with the ABC detection method (Vector Lab MI, USA) was performed for light microscope analysis. The analysis was conducted with a computer-assisted system (Image-Pro Plus, Media Cybernetics, L.P. Del Mar, CA, USA) connected by a CCD camera to Optiphot 2 microscope (Nikon, Japan). The number of TH-positive neurons was counted in $1500 \mu^{2}$ from 7 SNc sections per hemisphere of each animal (Avila-Costa et al. 2004). The total number of TH-positive cells was calculated manually rostro-caudally through the SNc and ventral tegmental area (VTA) in adjacent sections. The SNc was delineated using a manually traced region of interest (ROI) at low magnification (X 4). The number of TH positive cells was calculated in both hemispheres at the level of third cranial nerve, within a $100 \mu \mathrm{m}$ X $100 \mu \mathrm{m}$ counting area at high magnification (X 40) only within this defined ROI. The level of the third cranial nerve provides a strong anatomical landmark where the SNc can be consistently delineated from the VTA as described elsewhere (Iravani et al. 2002; Bukhatwa et al. 2009; Sánchez-Betancourt et al. 2012). Although not a stereological technique, previous studies have shown that the 3rd nerve rootlets provide a reliable anatomical landmark at which the extent of cell loss is reflective of cell loss throughout the entire substantia nigra (Iravani et al. 2002; Sánchez-Betancourt et al. 2012). Moreover, manual cell counts assessed at the level of the third cranial nerve have been demonstrated to give equivalent results, not significantly different to that obtained from unbiased stereological estimates at the same level using an optical fractionator probe design (Chan et al. 2010; Iravani et al. 2002; Sánchez-Betancourt et al. 2012).

Golgi Method

Blocks from the striatum were processed for the rapid Golgi method and cut into $90 \mu \mathrm{m}$ thick sections (Valverde, 1970). The histological analysis consisted in counting the number of dendritic spines in a $10 \mu \mathrm{m}$ long section from 5 secondary dendrites from 10 medium- sized spiny neurons per group in both hemispheres (Avila-Costa et al. 2004). 


\section{Statistical Analysis:-}

Data were analyzed using one-way analysis of variance (ANOVA) and Tukey's posthoc test, using the statistical software package GraphPad Prism version 7.0 for Mac (Graph Pad Software, San Diego, CA, USA). A P-value of 0.05 was selected as the threshold of statistical significance. Error bars represent \pm SEM.

\section{Results:-}

\section{Rotarod}

The rotarod test was divided into three stages: pre-exposure, three and six months of inhalation to determine progressive damage (Fig. 2A). The results show that there was a progressive decrease in the permanence in the rod directly proportional to the months of exposure. Between the pre-exposure stage vs. three months, there was an evident decrease in the rod permanence at revolutions 20 and 25 , decreasing by $46 \%$ and $8 \%$, respectively. However, at six months of $\mathrm{Mn}$ exposure, the decrease in the rod permanence was overwhelming in the five revolutions evaluated $(5,10,15,20$ and $25 \mathrm{rpm})$ the rod permanence loss was between 70 and $90 \%$. Figure $2 \mathrm{~A}$ shows the performance in the test.

The animals were analyzed at three months (when D3 agonist infusion pump life was terminated) and two months (no pump) after a total of five months to determine whether the recovery was progressive and permanent and compared to animals that did not receive treatment. We observed that at three months of treatment the animals still did not fully recover the motor ability to remain in the rotarod, an activity that was determined by the permanence time in the rod; but two months later (five-month total) and without treatment the animals recovered their activity, being significant the recovery in all revolutions evaluated, this was comparable to their activity in the pre-exposure stage (Fig. 2B).
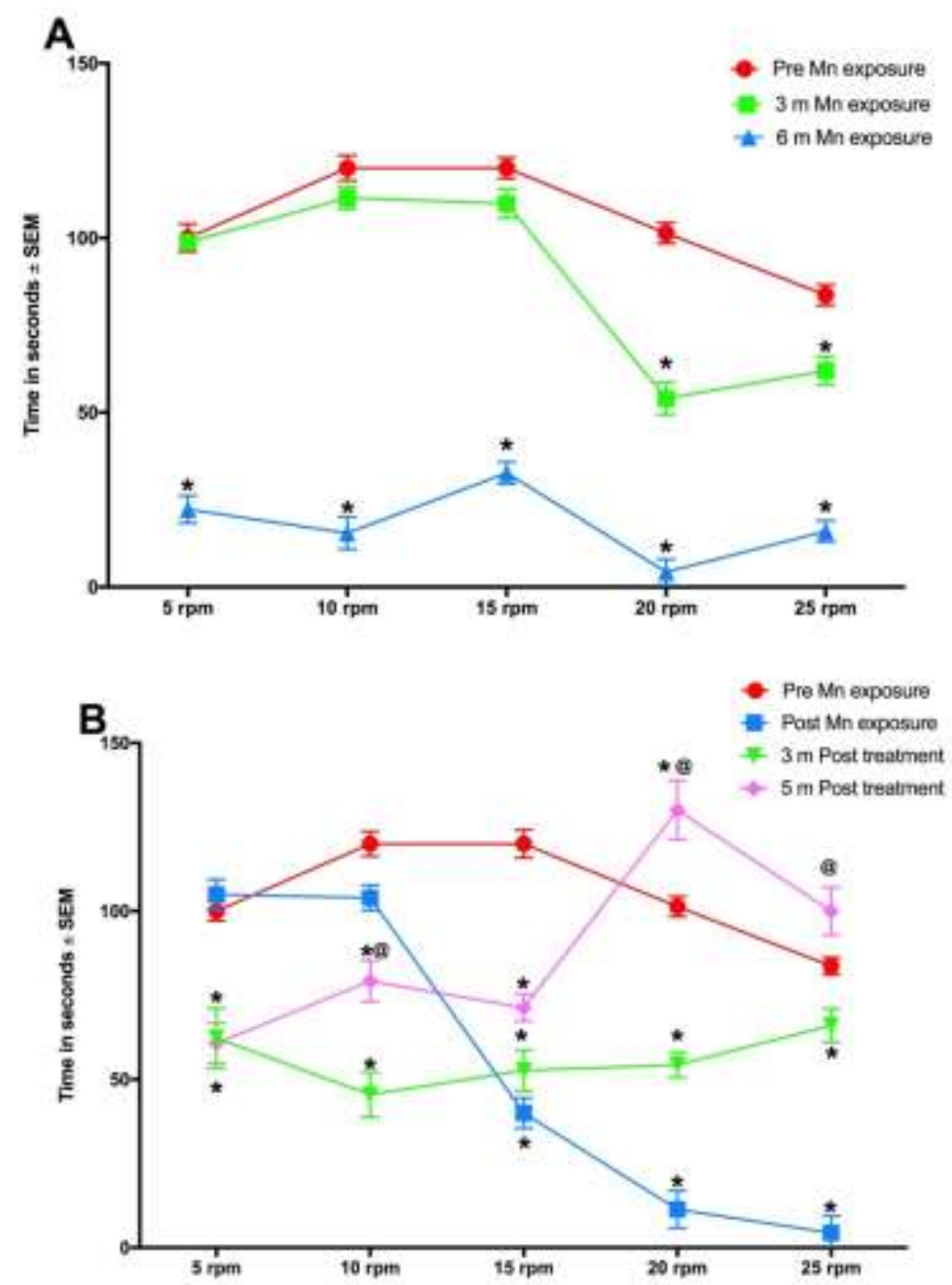
Figure 2:- Motor coordination (rotarod test). A Mn mixture inhalation reduced the time spent on the rod; after three months of inhalation the exposed rats spent less time on the rod, but after six months, the motor deterioration was more evident, the animals fall from the rod almost immediately. In contrast, in $\mathbf{B}$ it is observed that 7-OHDPAT/BDNF-transfection co-treatment improve the time on the rod, mainly after five months post treatment. Oneway ANOVA $* \mathrm{P}<0.05$ compared to control evaluation (Pre Mn exposure); @ $\mathrm{P}<0.05$ five months post treatment vs. three months post treatment, Mn saline-treated and Mn "recovery" groups. (Tukey multiple comparison posttests). The data are given as the mean \pm SEM ( $n=6$ rats/group).

\section{Open Field}

Motor activity progressive deterioration of the Mn-exposed group during its active phase was determined from the distance-traveled log. Mn-exposed animals decreased their exploratory activity directly proportional to the exposure time (three and six months) compared to the pre-exposure stage. Before Mn inhalation, the animals traveled time mean was of $5875 \mathrm{~cm}$ in $20 \mathrm{~min}$ (this value was taken as 100\%), at three months of exposure a significant decrease was observed, on average, each animal traveled $3250 \mathrm{~cm}$ which is equivalent to a $45 \%$ reduction; however, at six months, the decrease was more evident, corresponding to $66 \%$, traveling $1985.83 \mathrm{~cm}$ in $20 \mathrm{~min}$ (Fig. 3A). It was determined that motor activity recovery is trophic because it persisted even when the animals were no longer cotreated. At the end of three months, co-treatment showed a significant tendency of the animals to recover, two months after (without pump), the animals significantly recovered their exploratory activity (Fig. 3A). We also determined the speed of walking to assess bradykinesia, ambulation, and frequency of rearing during the first ten minutes and the freezing behavior. As seen in figure 3B Mn mixture inhalation, after three and six months induced bradykinesia, which was reverted with the co-treatment. Also, the rearing decrease (Fig. 3C) and freezing behavior increase (Fig. 3D), both behaviors statistically improved with the co-treatment (Figs. 3C and 3D).
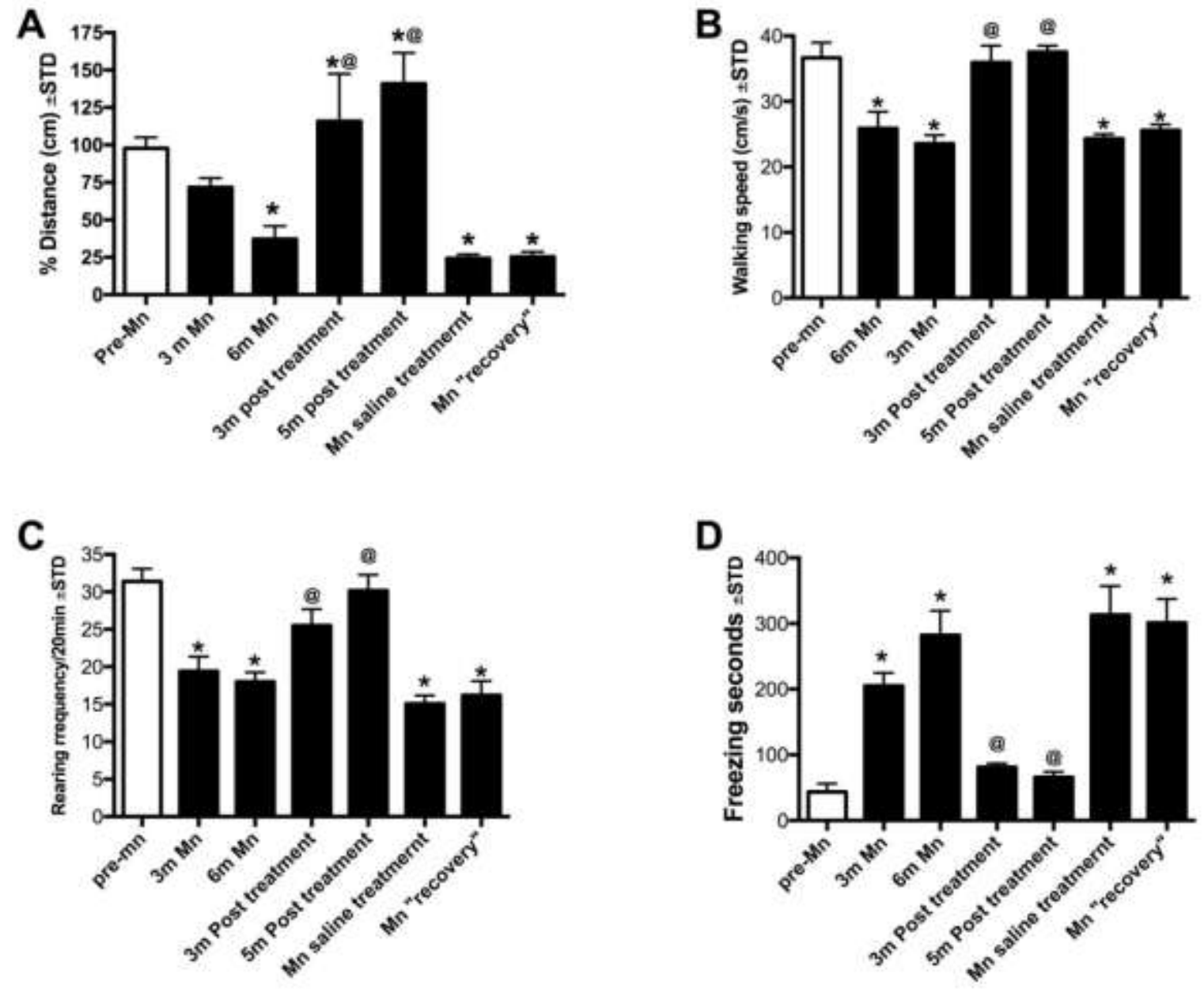
Figure 3:- Open Field Performance. Mn-mixture inhalation reduced the ambulatory activity evaluated by the distance traveled (A), induced bradykinesia $(\mathbf{B})$, reduced the rearing frequency $(\mathbf{C})$ and increased the freezing time (D) in the open field. It is evident that with the co-treatment, the animals significantly improve their behavior being more evident after five months post-treatment. One-way ANOVA *P $<0.05$ compared to control evaluation (Pre Mn exposure); @ P< 0.05 three and five months post-treatment vs. Mn saline-treated and Mn "recovery" groups. (Tukey multiple comparison posttests). The data are given as the mean \pm SEM ( $\mathrm{n}=6$ rats/group).

\section{Cytological Analysis:-}

$\mathrm{TH}^{+}$Nigral Cells Recovery

Bilateral counting of $\mathrm{TH}^{+}$neurons was performed throughout the $\mathrm{SNc}$ to determine if the co-treatment regained or maintained the number of neurons. These data were compared with the control, saline-treated and Mn-exposed without treatment ("recovery") groups. After Mn mixture exposure, there was a significant decrease in the number of TH+ neurons of $66.65 \%$. When counting the remaining neurons in the tissue of animals with the co-treatment of 7-OH-DPAT and BDNF (5 months post-treatment) a 100\% recovery was obtained in comparison with the control, saline-treated and "recovery" groups, determining that there were no significant differences between control and cotreated groups. This is shown in Figures 4 and 5.

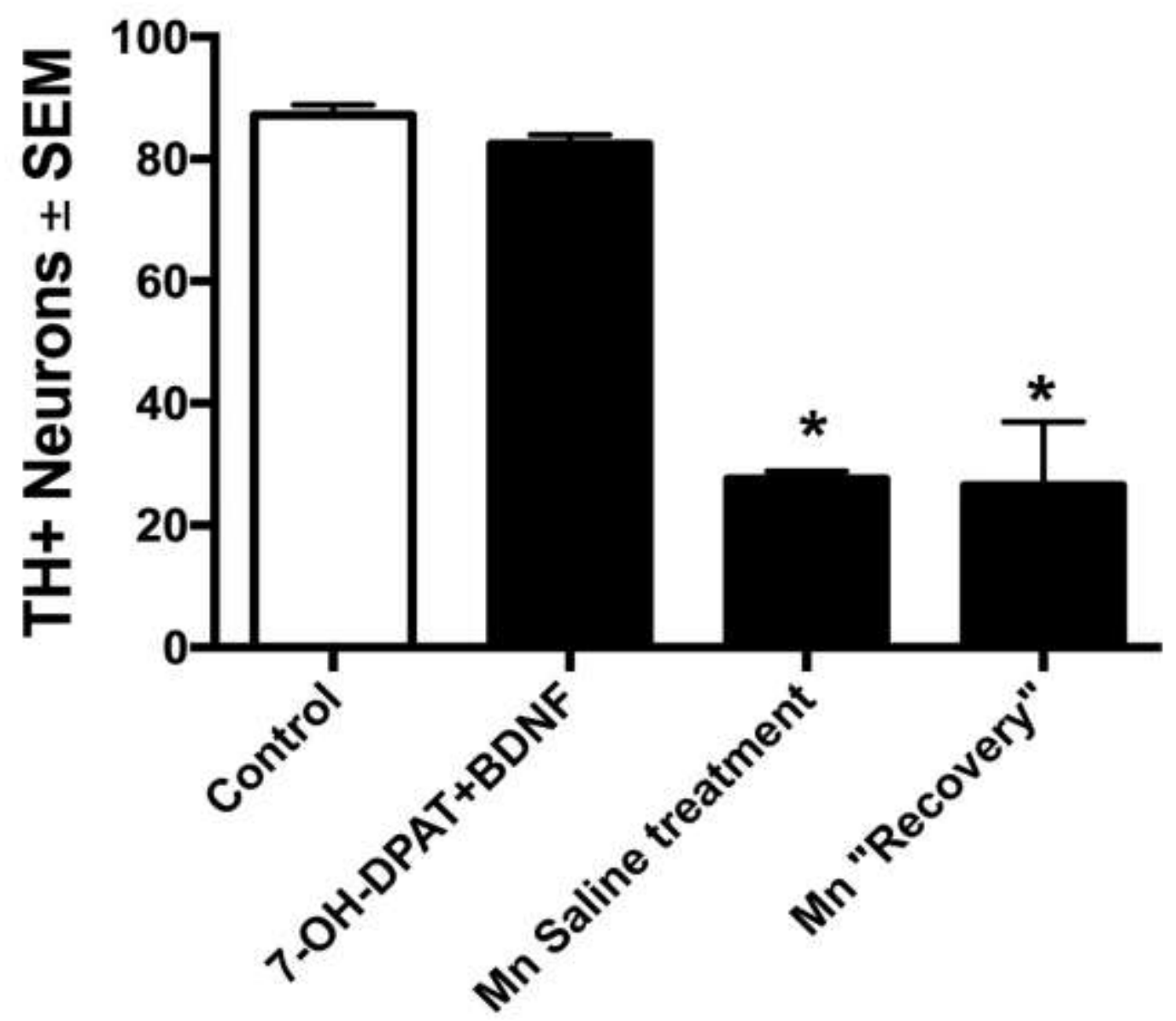

Figure 4:- Number of SNc TH+ neurons. It is evident than Mn inhalation drastically destroyed the dopaminergic neurons. However, the combined treatment recovered these neurons. One-way ANOVA $* \mathrm{P}<0.05$ compared with control evaluation (Pre Mn exposure) and co-treated groups. (Tukey multiple comparison posttests). The data are given as the mean \pm SEM ( $n=6$ rats/group). 


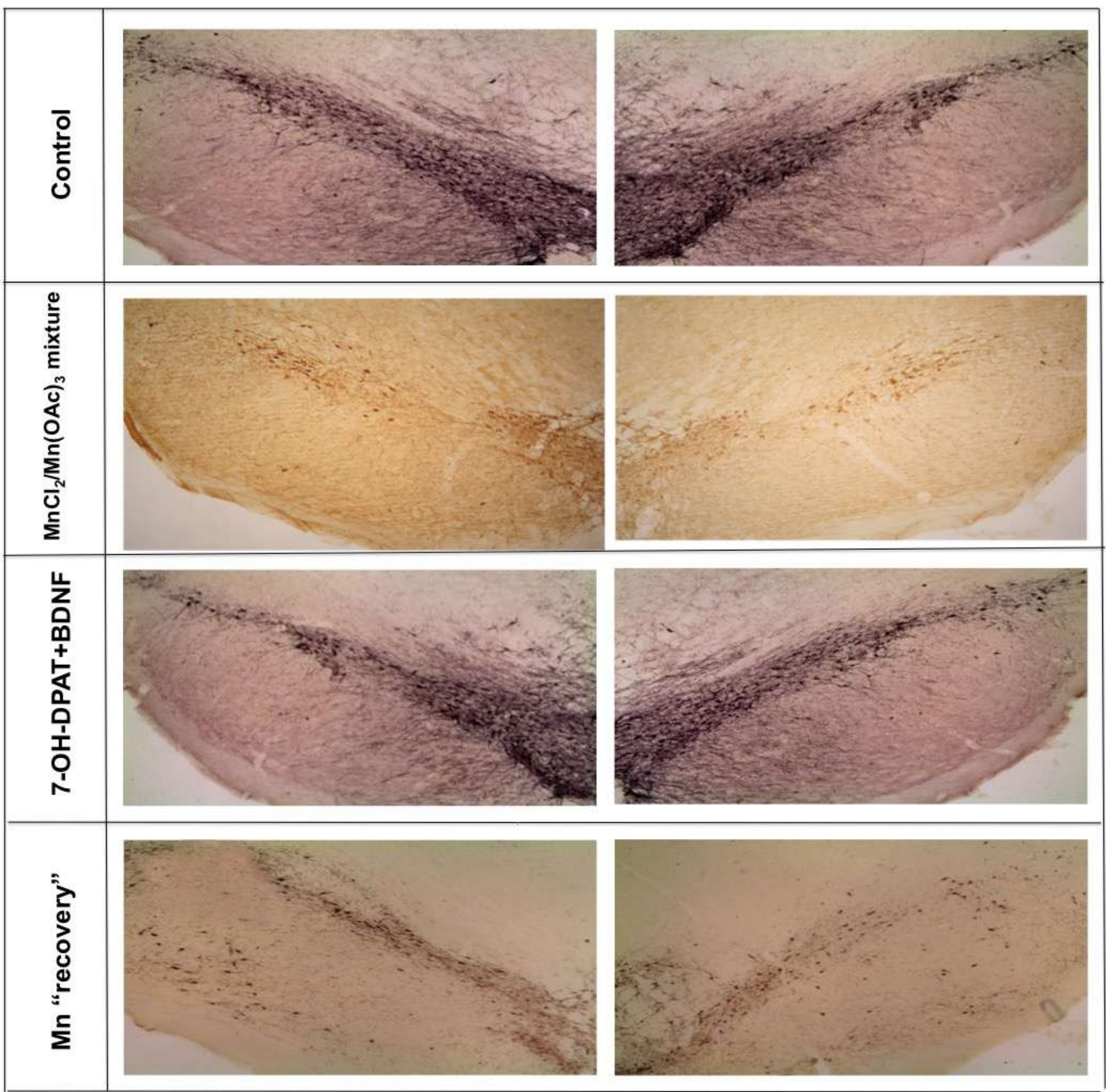

Figure 5:- SNc $\mathbf{T H}^{+}$neurons. Representative micrographs of TH-immunostained from coronal sections containing the SNc of the analyzed groups. The remaining dopaminergic neuronal bodies are observed in the animals of each experimental group $(40,000 \mathrm{X})$.

\section{Dendritic Spines}

Dendritic spine count was performed on neostriatal medium-sized spiny neurons to determine if the co-treatment recovers the number of dendritic spines. These data were compared with the control and Mn-exposed saline-treated and Mn-exposed "recovery" groups. The Mn model significantly reduced the number of spines of the medium-sized spiny neurons of the striatum (Figs. 6 and 7). The D3 agonist associated with the BDNF gene fully recovered the 
spines. In contrast, both, Mn-exposed group ("recovery") and Mn-exposed saline treated showed a significant dendritic spine loss (Figs. 6 and 7).

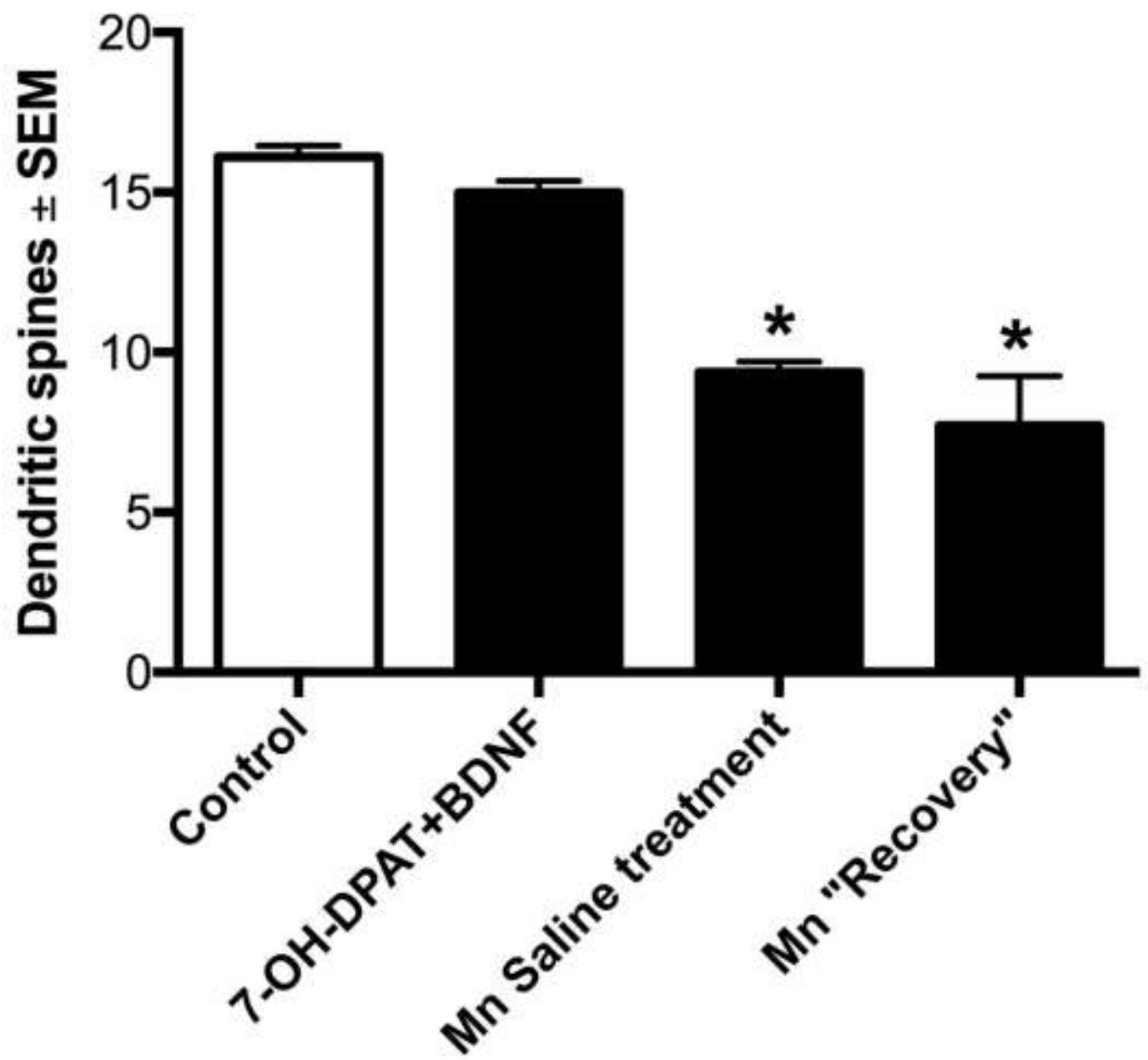

Figure 6:- Number of striatal medium-sized spiny neurons dendritic spines. It is notorious the dendritic spine loss after Mn compounds inhalation. However, the 7-OH-DPAT/BDNF-infusion co-treatment significantly restored the dendritic spines density. One-way ANOVA *P< 0.05 compared with control evaluation (Pre Mn exposure) and co-treated groups. (Tukey multiple comparison posttests). The data are given as the mean \pm SEM ( $n=6$ rats/group). 

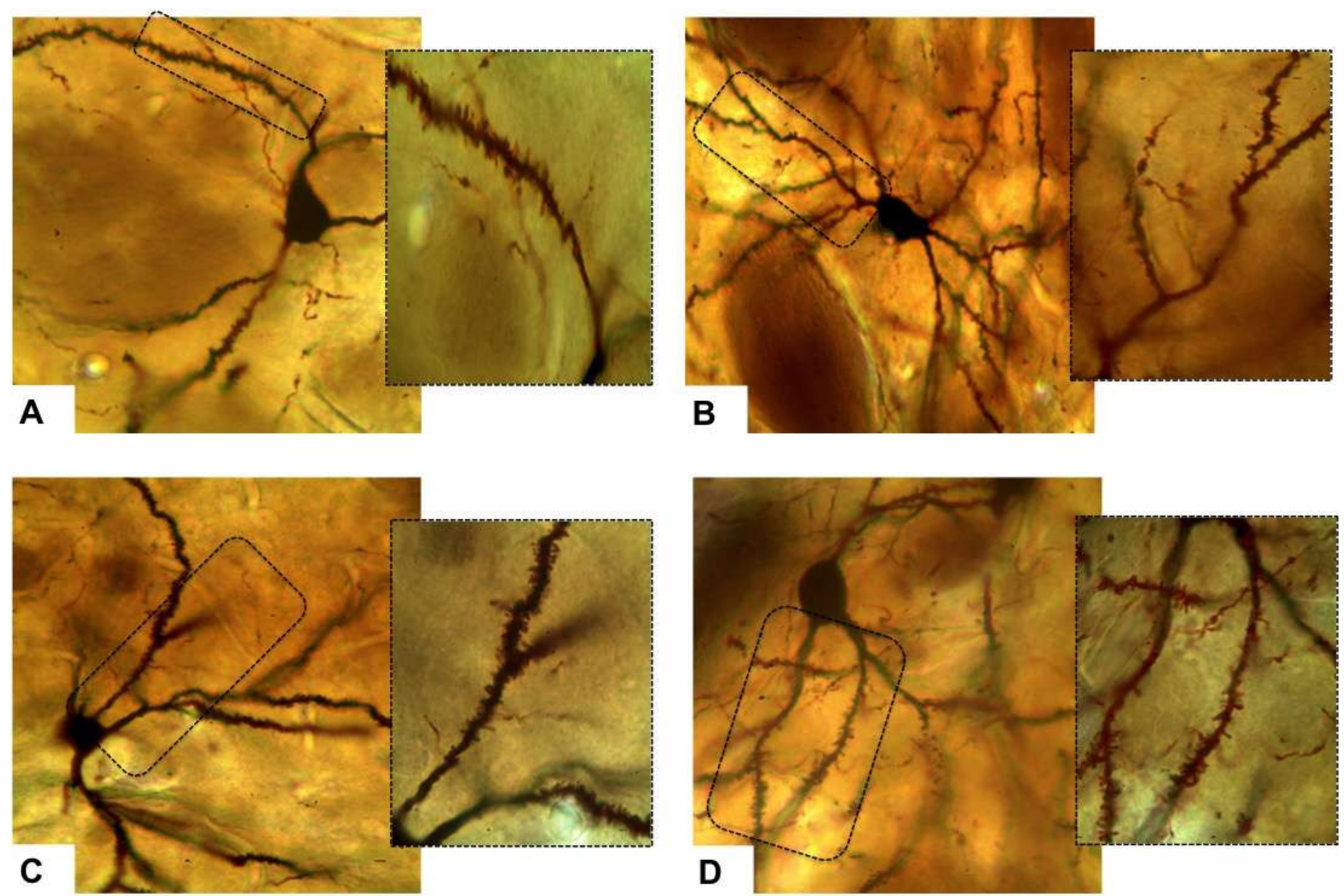

Figure 7:- Dendritic spine density. Photomicrographs of representative Golgi-stained medium-sized spiny neurons of the striatum with a representative box of dendritic spine densities from the control group (A), Mn saline-treated (B), 7-OHDPAT/BDNF co-treated $(\mathbf{C})$, and Mn- "recovery" group (D). Mn compounds inhalation induced a marked decrease in the total number of spines. In contrast, the combined treatment induced a well-preserved dendritic spine ensity (magnification, $40 \mathrm{X}$ and $100 \mathrm{X}$ ).

\section{Discussion:-}

To date, the most frequently used treatments for PD, such as L-DOPA, deep stimulation or transplants have failed to stop the progression or induce DA neurons regeneration; in the case of L-DOPA, after a long time of administration it turns out to be toxic since it accelerates the disease progression and induce symptoms like dyskinesia, which become more incapacitating than the disease (Ahlskog et al. 2001). Recent approaches have reported that stimulation of neurogenic niches could replace at least part of the loss of the original SNc neurons and re-innervate the motor circuit, restoring the balance between basal ganglia direct and indirect pathways (Van Kampen and Eckman, 2006; Razgado-Hernandez et al. 2015).

Continuous and chronic administration of the D3 agonist associated with BDNF gene transfection showed great recovery of motor activity in the rotarod and open field tests, as well as the regeneration or neuroprotection of SNc DA neurons and striatal dendritic spines of 6-OHDA unilaterally lesioned animals (Razgado-Hernandez et al. 2015). Our results showed that in the rotarod test the animals that inhaled the Mn mixture and received the co-treatment, partially recovered their performance in the motor activity, remaining longer at the speed of 20 rpm at two months 
after the co-treatment was applied; these data are consistent with those reported by Hernandez-Chan et al. (2015), where animals at 12 weeks of 7-OH-DPAT and BDNF co-treatment, have improved motor performance in the rotarod test (10, 15 and $20 \mathrm{rpm}$ ) compared to the 6-OHDA lesion phase. And with Razgado et al. (2015) whose experimental animals (intrastriatally 6-OHDA lesioned) received the co-treatment 7-OH-DPAT and BDNF, presented recovery in the rotarod permanence time at 6.5 months of co-treatment.

Our open field test results showed that recovery is relative to the co-treatment administration time; at five months, the animals recover their exploratory activity $100 \%$, increase the movement speed through the field, exploring it in greater quantity and presenting few immobility periods, so it is presumed that bradykinesia symptom is eliminated. This data is consistent with the study by Razgado et al. (2015), where rats with 6-OHDA lesion and that received the 7-OH-DPAT and BDNF co-treatment obtained similar results. It is important to mention that the treatment is optimal five months later after started, considering that the co-treatment is only present three months and two months after it is withdrawn, this means that the treatment has a trophic function because its effect remains. Once it was determined that with the co-treatment the motor alterations significantly improve, with the cytological analysis it was analyzed whether this motor recovery was related to the DA system restoration. After six months of Mn mixture inhalation there was a loss of $66.65 \%$ of SNc DA neurons; and five months after, the co-treatment administration the neuronal recovery was $100 \%$ comparing to Mn-untreated and the control groups; this is similar with the data shown by Razgado et al. (2015) in rats with striatal 6-OHDA lesion and 7-OH-DPAT and BDNF cotreated, which obtained a $95 \%$ recovery.

It should be emphasized that the DA neurons recovery did not produce dyskinetic movements as reported in DA transplants to the striatum (Politis et al. 2011; Ma et al. 2011; Shin et al. 2014), implying that, in our case, the striatal innervation functional recovery was not accompanied by an extreme striatal DA innervation.

It is important to mention that the new generation neurons must be functional; it is known that the SNc DA neurons innervate the striatum, which is the main responsible for motor activity, that is why the nigrostriatal pathway recovery is indispensable; the rescue of the striatal dendritic spines is a collateral data of DA system recovery (Yao et al. 2008; Fasano et al. 2013; Toy et al. 2014) and is a necessary condition because it is the place where the DA synaptic contacts take place (Day et al. 2006); we observe that after Mn inhalation the dendritic spine loss is $27.1 \%$ and after the co-treatment the recovery is $100 \%$, presenting values very similar to the control group. Therefore, the complete dendritic spines recovery brought about by the D3 receptors activation associated with the BDNF transfection may explain the motor behavior recovery observed in the present work. This fact is in line with the statement that the striatal dopamine grafts efficacy also needs the dendritic spines preservation or recovery (Soderstrom et al. 2010).

The DA neurons regeneration and functionality is attributed to the synergistic relationship between the D3 receptor and BDNF. It seems that BDNF is responsible for the D3 receptors expression during embryonic development and for maintaining their expression in the adult brain (Sokoloff et al. 2002); the D3 receptor is synthesized by the SNc DA neurons (Howells et al. 2000), and is responsible for their neurogenic activity, it has been reported that when the D3 receptor is selectively stimulated, the nigrostriatal circuit is restored (Van Kampen et al. 2004; Van Kampen and Eckman, 2006), which is reflected in the motor performance (Ouagazzal and Creese, 2000; Van Kampen and Eckman, 2006).

\section{Conclusion:-}

Our findings provide evidence that the continuous D3 receptor activation associated with the BDNF gene non-viral transfection to the SNc DA neurons induce a significant and persistent motor behavior recovery in a bilateral PD rat model. The behavioral recovery is in association with the increased $\mathrm{TH}^{+}$neurons of a well-reinnervated striatum, evidenced by the dendritic spines recovery of the striatal medium-sized spiny neurons. The pharmacological effect is improbable to explain the functional recovery since the motor behavior improvement continued two months after treatment was finished, suggesting a trophic effect. This combined treatment appears to be a favorable approach for DA cells recovering in this PD experimental model, which in our opinion is a middle PD stage because we found $66.65 \%$ cell death, and, since it has been reported that D3 receptors decrease with the PD progression (Szabolcs et al. 2012; Rangel-Barajas et al. 2015), thus this kind of treatment should be given at initial or middle stages of the disease. Moreover, we assure that the inhalation of $\mathrm{MnCl}_{2} / \mathrm{Mn}(\mathrm{OAc})_{3}$ mixture is an appropriate PD model, since it provides similar behavioral and morphological changes to those observed in PD patients contributing as a 
convenient experimental model for the study of this neurodegenerative disease and the animals recover with a DA treatment.

\section{Acknowledgments:-}

This work was supported by the research grants from PAPIIT-DGAPA - UNAM PAPIIT-DGAPA IN215114, IN219617 and PAPCA-Iztacala UNAM 2016-13.

The authors thank Jesus Espinosa Villanueva, Veronica Rodríguez Mata and Patricia Aley Medina for their excellent photographic and technical assistance.

\section{References:-}

1. Ahlskog JE, Muenter MD (2001) Frequency of levodopa-related dyskinesias and motor fluctuations as estimated from the cumulative literature. Mov Disord 16: 448-458.

2. Alexander GE (2004) Biology of Parkinson's disease: pathogenesis and pathophysiology of a multisystem neurodegenerative disorder. Dialogues in Clin Neurosci 6: 259-280.

3. Alvarez-Maya I, Navarro-Quiroga I, Meraz-Rios MA, Aceves J, Martinez-Fong D (2001) In vivo gene transfer to dopamine neurons of rat substantia nigra via the high-affinity neurotensin receptor. Mol Med 7: 186-192.

4. Avila-Costa MR, Montiel Flores E, Colin-Barenque L, Ordoñez JL, Gutiérrez AL, Niño-Cabrera HG, MussaliGalante P, Fortoul TI (2004) Nigrostriatal modifications after vanadium inhalation: an immunocytochemical and cytological approach. Neurochem Res 29: 1365-1369.

5. Benraiss A, Chmielnicki E, Lerner K, Roh D, Goldman SA (2001) Adenoviral brain-derived neurotrophic factor induces both neostriatal and olfactory neuronal recruitment from endogenous progenitor cells in the adult forebrain. J Neurosci 21: 6718-6731.

6. Betarbet R, Sherer TB, Greenamyre JT (2002) Animal models of Parkinson's disease. BioEssays 24: 308-318.

7. Blochl A, Sirrenberg C (1996) Neurotrophins stimulate the release of dopamine from rat mesencephalic neurons via Trk and p75Lntr receptors. J Biol Chem 271: 21100-21107.

8. Bukhatwa S, Iravani MM, Zeng BY, Cooper JD, Rose S, Jenner P (2009) An immunohistochemical and stereological analysis of PSI-induced nigral neuronal degeneration in the rat. J Neurochem 109: 52-59.

9. Casarrubea M, Sorbera F, Crescimanno G (2006) Effects of 7-OH-DPAT and U 99194 on the behavioral response to hot plate test, in rats. Physiol Behav 89: 552-562.

10. Chan H, Paur H, Vernon AC, Zabarsky V, Datla KP, Croucher MJ, Dexter DT (2010) Neuroprotection and Functional Recovery Associated with Decreased Microglial Activation Following Selective Activation of mGluR2/3 Receptors in a Rodent Model of Parkinson's Disease Parkinsons Dis 2010:190450.

11. Chio CL, Lajiness ME, Huff RM (1994) Activation of heterologously expressed D3 dopamine receptors: comparison with D2 dopamine receptors. Mol Pharmacol 45: 51-60.

12. Collo G, Zanetti S, Missale C, Spano P (2008) Dopamine D3 receptor-preferring agonists increase dendrite arborization of mesencephalic dopaminergic neurons via extracellular signal-regulated kinase phosphorylation. Eur J Neurosci 28: 1231-1240.

13. Connor B, Dragunow M (1998) The role of neuronal growth factors in neurodegenerative disorders of the human brain. Brain Res Rev, 27: 1-39.

14. Cummins G, Barker RA, (2012) What is the most promising treatment for Parkinson's disease: genes, cells, growth factors or none of the above? Regen Med, 7: 617-621.

15. Day M, Wang Z, Ding J, An X, Ingham CA, Shering AF, Wokosin D, Ilijic E, et al. (2006) Selective elimination of glutamatergic synapses on striatopallidal neurons in Parkinson disease models. Nat Neurosci 9: 251-259.

16. Diaz J, Ridray S, Mignon V, Griffon N, Schwartz JC, Sokoloff P (1997) Selective expression of dopamine D3 receptor mRNA in proliferative zones during embryonic development of the rat brain. J Neurosci 17: 42824292.

17. Du F, Li R, Huang Y, Li X, Le W (2005) Dopamine D3 receptor-preferring agonists induce neurotrophic effects on mesencephalic dopamine neurons. Eur J Neurosci 22: 2422-2430.

18. Emborg ME (2004) Evaluation of animal models of Parkinson's disease for neuroprotective strategies, J Neurosci Methods 139: 121-143.

19. Fasano C, Bourque MJ, Lapointe G, Leo D, D. Thibault, M. Haber, Kortleven C, DesGroseillers L, et al. (2013) Dopamine facilitates dendritic spine formation by cultured striatal medium spiny neurons through both D1 and D2 dopamine receptors. Neuropharmacology 67: 432-443. 
20. Fritsch B, Reis J, Martinowich K, Schambra HM, Ji Y, Cohen LG, Lu B (2010) Direct current stimulation promotes BDNF-dependent synaptic plasticity: potential implications for motor learning. Neuron 66: 198-204.

21. Goggi J, Pullar IA, Carney SL, Bradford HF (2002) Modulation of neurotransmitter release induced by brainderived neurotrophic factor in rat brain striatal slices in vitro. Brain Res, 941: 34-42.

22. Gonzalez-Barrios JA, Lindahl M, Bannon MJ, Anaya-Martinez V, Flores G, Navarro-Quiroga I, Trudeau LE, Aceves J, et al. (2006) Neurotensin polyplex as an efficient carrier for delivering the human GDNF gene into nigral dopamine neurons of hemiparkinsonian rats. Mol Ther 14: 857-865.

23. Griffon N, Pilon C, Sautel F, Schwartz JC, Sokoloff P (1997) Two intracellular signaling pathways for the dopamine D3 receptor: opposite and synergistic interactions with cyclic AMP. J Neurochem 68: 1-9.

24. Guillin O, Diaz J, Carroll P, Griffon N, Schwartz JC, Sokoloff P (2001) BDNF controls dopamine D3 receptor expression and triggers behavioural sensitization. Nature 411: 86-89.

25. Hernandez-Baltazar D, Martinez-Fong D, Trudeau LE (2012) Optimizing NTS-polyplex as a tool for gene transfer to cultured dopamine neurons. PloS one 7: e51341.

26. Hernandez-Chan NG, Bannon MJ, Orozco-Barrios CE, Escobedo L, Zamudio S, De la Cruz F, Gongora-Alfaro JL, Armendariz-Borunda J, et al. (2015) Neurotensin-polyplex-mediated brain-derived neurotrophic factor gene delivery into nigral dopamine neurons prevents nigrostriatal degeneration in a rat model of early Parkinson's disease. J Biomed Sci, 22: 59.

27. Howells DW, Porritt MJ, Wong JY, Batchelor PE, Kalnins R, Hughes AJ, Donnan GA (2000) Reduced BDNF mRNA expression in the Parkinson's disease substantia nigra. Exp Neurol 166: 127-135.

28. Hyman C, Hofer M, Barde YA, Juhasz M, Yancopoulos GD, Squinto SP, Lindsay RM (1991) BDNF is a neurotrophic factor for dopaminergic neurons of the substantia nigra, Nature, 350: 230-232.

29. Im SH, Yu JH, Park ES, Lee JE, Kim HO, Park KI, Kim GW, Park CI, et al. (2010) Induction of striatal neurogenesis enhances functional recovery in an adult animal model of neonatal hypoxic-ischemic brain injury. Neuroscience 169: 259-268.

30. Iravani MM, Kashefi K, Mander P, Rose S, Jenner P (2002) Involvement of inducible nitric oxide synthase in inflammation-induced dopaminergic neurodegeneration. Neuroscience 110: 49-58.

31. Ma Y, Peng S, Dhawan V, Eidelberg D (2011) Dopamine cell transplantation in Parkinson's disease: challenge and perspective. Br Med Bull 100: 173-189.

32. Martin WR (2011) Fuming over Parkinson disease: are welders at risk?, Neurology 76: 1286-1287.

33. Martinez-Fong D, Bannon MJ, Trudeau L-É, Gonzalez-Barrios JA, Arango-Rodriguez ML, Hernandez-Chan NG, Reyes-Corona D, Armendáriz-Borunda J, et al. (2012) NTS-Polyplex: A potential nanocarrier for neurotrophic therapy of Parkinson's disease. Nanomedicine 8: 1052-1069.

34. Merlo S, Canonico PL, Sortino MA (2011) Distinct effects of pramipexole on the proliferation of adult mouse sub-ventricular zone-derived cells and the appearance of a neuronal phenotype. Neuropharmacology 60: 892900.

35. Mogi M, Togari A, Kondo T, Mizuno Y, Komure O, Kuno S, Ichinose H, Nagatsu T (1999) Brain-derived growth factor and nerve growth factor concentrations are decreased in the substantia nigra in Parkinson's disease. Neurosci Lett, 270: 45-48.

36. Naskar A, Manivasagam T, Chakraborty J, Singh R, Thomas B, Dhanasekaran M, Mohanakumar KP (2013) Melatonin synergizes with low doses of L-DOPA to improve dendritic spine density in the mouse striatum in experimental Parkinsonism. J Pineal Res 55: 304-301.

37. Ouagazzal AM, Creese I (2000) Intra-accumbens infusion of D-3 receptor agonists reduces spontaneous and dopamine-induced locomotion. Pharmacol Biochem Behav 67: 637-645.

38. Ordóñez-Librado JL, Anaya-Martínez V, Gutiérrez-Valdez AL, Colín-Barenque L, Montiel-Flores E, AvilaCosta MR (2010a) Manganese inhalation as a Parkinson disease model. Parkinsons Dis 2011: 612989.

39. Ordoñez-Librado JL, Anaya-Martínez V, Gutierrez-Valdez AL, Montiel-Flores E, Corona DR, Martinez-Fong D, Avila-Costa MR (2010b) L-DOPA treatment reverses the motor alterations induced by manganese exposure as a Parkinson disease experimental model. Neurosci Lett 471: 79-82.

40. Ordoñez-Librado JL, Gutierrez-Valdez AL, Colín-Barenque L, Anaya-Martínez V, Díaz-Bech P, Avila-Costa MR (2008) Inhalation of divalent and trivalent manganese mixture induces a Parkinson's disease model: immunocytochemical and behavioral evidences. Neuroscience 155: 7-16.

41. Paxinos G, Watson C (2005) The rat brain atlas in stereotaxic coordinates, Elsevier Academic Press, New York.

42. Pilon C, Levesque D, Dimitriadou V, Griffon N, Martres MP, Schwartz JC, Sokoloff P (1994) Functional coupling of the human dopamine D3 receptor in a transfected NG 108-15 neuroblastoma-glioma hybrid cell line. Eur J Pharmacol 268: 129-139. 
43. Politis M, Oertel WH, Wu K, Quinn NP, Pogarell O, Brooks DJ, Björklund A, Lindvall O, et al. (2011) Graftinduced dyskinesias in Parkinson's disease: High striatal serotonin/dopamine transporter ratio. Mov Disor 26: 1997-2003.

44. Porritt M, Stanic D, Finkelstein D, Batchelor P, Lockhart S, Hughes A, Kalnins R, Howells D (2005) Dopaminergic innervation of the human striatum in Parkinson's disease. Mov Disors 20: 810-818.

45. Potashkin JA, Blume SR, Runkle NK (2010) Limitations of animal models of Parkinson's disease. Parkinsons Dis 2011: 658083.

46. Prut L, Belzung C (2003) The open field as a paradigm to measure the effects of drugs on anxiety-like behaviors: a review. Eur J Pharmacol 463: 3-33.

47. Rangel-Barajas C, Coronel I, Florán B. (2015) Dopamine Receptors and Neurodegeneration. Aging Dis 6: 349368.

48. Razgado-Hernandez LF, Espadas-Alvarez AJ, Reyna-Velazquez P, Sierra-Sanchez A, Anaya-Martínez V, Jimenez-Estrada I, Bannon MJ, Martinez-Fong D, et al. (2015) The transfection of BDNF to dopamine neurons potentiates the effect of dopamine D3 receptor agonist recovering the striatal innervation, dendritic spines and motor behavior in an aged rat model of Parkinson's disease PloS one, 10: e0117391.

49. Rozas G, Lopez-Martin E, Guerra MJ, Labandeira-Garcia JL (1998) The overall rod performance test in the MPTP-treated-mouse model of Parkinsonism. J Neurosci Methods 83: 165-175.

50. Sánchez-Betancourt J, Anaya-Martínez V, Gutiérrez-Valdez AL, Ordóñez-Librado JL, Montiel-Flores E, Espinosa-Villanueva J, Reynoso-Erazo L, Avila-Costa MR (2012) Manganese mixture inhalation is a reliable Parkinson disease model in rats. Neurotoxicology 33: 1346-1355.

51. Shin E, Lisci C, Tronci E, Fidalgo C, Stancampiano R, Björklund A, Carta M (2014) The anti-dyskinetic effect of dopamine receptor blockade is enhanced in parkinsonian rats following dopamine neuron transplantation. Neurobiol Dis 63: 233-240.

52. Smith Y, Wichmann T, Factor SA, DeLong MR (2012) Parkinson's disease therapeutics: new developments and challenges since the introduction of levodopa. Neuropsychopharmacology 37:213-46.

53. Soderstrom KE, O'Malley JA, Levine ND, Sortwell CE, Collier TJ, Steece-Collier K (2010) Impact of dendritic spine preservation in medium spiny neurons on dopamine graft efficacy and the expression of dyskinesias in parkinsonian rats. Eur J Neurosci 31: 478-490.

54. Sokoloff P, Guillin O, Diaz J, Carroll P, Griffon N (2002) Brain-derived neurotrophic factor controls dopamine D3 receptor expression: implications for neurodevelopmental psychiatric disorders. Neurotox Res, 4: 671-678.

55. Szabolcs F, Katalin N, Zhisheng J, Tibor H, Miklés P, Sean D, Pike VW, Halldin C, et al. (2012) The decrease of dopamine $\mathrm{D}_{2} / \mathrm{D}_{3}$ receptor densities in the putamen and nucleus caudatus goes parallel with maintained levels of $\mathrm{CB}_{1}$ cannabinoid receptors in Parkinson's disease: A preliminary autoradiographic study with the selective dopamine $\mathrm{D}_{2} / \mathrm{D}_{3}$ antagonist $\left[{ }^{3} \mathrm{H}\right]$ raclopride and the novel $\mathrm{CB}_{1}$ inverse agonist $\left.{ }^{125} \mathrm{I}\right] \mathrm{SD} 7015$. Brain Res Bull 87: 504-510.

56. Toy WA, Petzinger GM, Leyshon BJ, Akopian GK, Walsh JP, Hoffman MV, Vučković MG, Jakowec MW (2014) Treadmill exercise reverses dendritic spine loss in direct and indirect striatal medium spiny neurons in the 1-methyl-4-phenyl-1,2,3,6-tetrahydropyridine (MPTP) mouse model of Parkinson's disease. Neurobiol Dis 63: 201-209.

57. Valverde F (1970) The Golgi Method. A Tool for Comparative Structural Analyses, in: W.J.H. Nauta, S.O.E. Ebbesson (Eds.) Contemporary Research Methods in Neuroanatomy, Springer Berlin Heidelberg, pp. 12-31.

58. Van Kampen JM, Eckman CB (2006) Dopamine D3 receptor agonist delivery to a model of Parkinson's disease restores the nigrostriatal pathway and improves locomotor behavior. J Neurosci 26: 7272-7280.

59. Van Kampen JM, Hagg T, Robertson HA (2004) Induction of neurogenesis in the adult rat subventricular zone and neostriatum following dopamine D3 receptor stimulation. Eur J Neurosci 19: 2377-2387.

60. Venero JL, Beck KD, Hefti F (1994) 6-Hydroxydopamine lesions reduce BDNF mRNA levels in adult rat brain substantia nigra, Neuroreport, 5: 429-432.

61. Yao WD, Spealman RD, Zhang J (2008) Dopaminergic signaling in dendritic spines. Biochem Pharmacol 75: 2055-2069.

62. Yurek DM, Lu W, Hipkens S, Wiegand SJ (1996) BDNF enhances the functional reinnervation of the striatum by grafted fetal dopamine neurons. Exp Neurol 137: 105-118.

63. Zhou J, Bradford HF, Stern GM (1994a) The response of human and rat fetal ventral mesencephalon in culture to the brain-derived neurotrophic factor treatment. Brain Res 656: 147-156.

64. Zhou J, Bradford HF, Stern GM (1994b) The stimulatory effect of brain-derived neurotrophic factor on dopaminergic phenotype expression of embryonic rat cortical neurons in vitro. Brain Res Dev 81: 318-324. 